Copyright (C) 2014 by Academic Publishing House Researcher

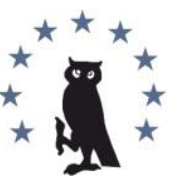

Published in the Russian Federation

European Researcher

Has been issued since 2010.

ISSN 2219-8229

E-ISSN 2224-0136

Vol. 82, No. 9-1, pp. 1611-1619, 2014

DOI: 10.13187/er.2014.82.1611

www.erjournal.ru

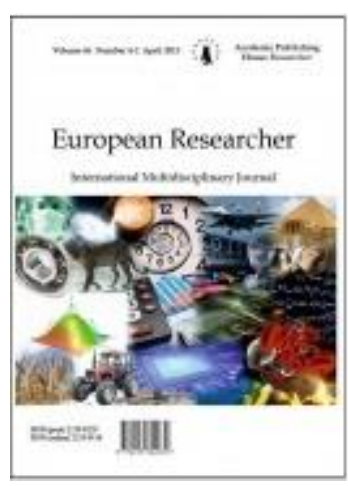

Technical Sciences

Технические науки

UDC 528; 004.9

\title{
Digital Simulation in the Geosciences
}

Alexandr A. Lobanov

Moscow State Technical University of Radio Engineering, Electronics and Automation MSTU,

Russian Federation

Ph.D., Associate Professor

E-mail: cvj2@mail.ru

\section{Abstract}

This article provides an analysis of methods for digital modeling in the area of Earth Sciences. The author illustrates the difference between digital modeling in radio communication and that in the area of Earth Sciences. The article examines the integration aspect of digital models, demonstrates the advantages of digital over analog models, and illustrates that digital models are discrete. The author outlines the characteristics of digital modeling and illustrates the logical structure of digital models.

Keywords: Information; knowledge; geoinformatics; model; modeling; digital simulation; numerical models.

\section{Введение}

Современные формы представления и обработки информации связаны с цифровыми методами и цифровыми моделями. По мере развития систем коммуникаций и вычислительной техники, а также перевода различных видов информации в дискретную форму появились термины «цифровые данные» [1], «цифровая информация» [2], «цифровые технологии» [3], «цифровые методы», «цифровые системы» [4]. Появление новой формы представления информации вызвали совместные процессы глобализации и информатизации [5].

Для обработки информации в информационных системах и $\mathrm{c}$ помощью информационных технологий все виды информации переводят в цифровую форму. Таким образом, цифровая форма представления информации интегрирует (объединяет) различные виды информации и создает возможность их совместной обработки. Однако существует различие в применении цифровых методов и моделей в разных научных направлениях. В области наук о Земле эта специфика проявляется наиболее ярко [6], что делает актуальным исследование цифрового моделирования в этом направлении. 
Развитие и использование цифровых методов. Развитие вычислительной техники, сопровождаемое снижением ее стоимости и увеличением мощности вычислений, предопределило широкое внедрение цифровых технологий и систем во многие сферы человеческой деятельности.

Следует отметить, что цифровые методы и цифровое моделирование появились в радиотехнике и электронике задолго до их появления в области наук о Земле [7]. Основоположником цифрового моделирования следует считать К.Э. Шеннона, что, например, показано в [8]. Поэтому альтернативой цифровым моделям являются аналоговые модели.

Цифровые методы и системы могут использоваться не только в компьютерных технологиях, но и в других областях (цифровое телевидение, цифровая телефонная связь, цифровая фотография и др.) в этих системах цифровой означает необходимость работы с дискретной информацией. Следует отметить свойства цифрового моделирования.

1. Первое свойство это высокая помехозащищенность. Дискретный сигнал или модель более систематизирована и ее легче фильтровать и восстанавливать. При этом помехозащищенность дискретного сигнала на порядки выше, чем аналогового.

2 Высокая информативность, сопоставимая с информативностью аналоговой модели (исходного объекта). Теорема отсчетов Хартли-Найквиста-Шеннона-Котельникова (известная в России просто как теорема Котельникова) гарантирует, при определенных условиях дискретизации (цифрования), сохранение информативности в дискретном сигнале. Именно это дает возможность построения на основе цифровой модели вторичную аналоговую модель.

3. Дискретная модель требует на порядки меньше памяти на машинных носителях, чем аналоговая.

4. Дискретная (цифровая) модель (сигнал) может передаваться с большей скоростью по сетям телекоммуникаций. Большая скорость передачи и обработки важной свойство цифровых методов. Для компьютеров это выражается в более высокой скорости обработки информации.

5. Дискретные сигналы можно обрабатывать с помощью любых алгоритмов. Универсальность обработки еще одно свойство цифровых методов и моделей.

6. Качество передачи информации приводит к более высокому качеству восстановления цифровых сигналов. Это определило их широкое применение в телевидении, радиовещании и телефонной связи. а также в спутниковых радионавигационных системах.

7. Дискретные сигналы требуют меньшую ширину полосы пропускания, для передачи информации, чем аналоговые.

8. Методы цифровой обработки изображений обычно являются более точными, надёжными, гибкими и простыми в реализации, нежели аналоговые методы. В цифровой обработке изображений широко применяется специализированное оборудование, такое как процессоры с конвейерной обработкой инструкций и многопроцессорные системы. В особенной мере это касается систем обработки видео.

Цифровые методы обработки пространственной информации. Цифровые системы и технологии обработки пространственной информации делятся на два больших класса: обработка двумерной геоинформации и обработка трехмерной геоинформации. В свою очередь, обработка двухмерной геоинформации разделяется на два подкласса: обработку изображений и обработку плановых координат.

Разница между этими подклассами в том, что для обработки изображений используют, как правило, целочисленные координаты, а для обработки плановых координат (геодезических или фотограмметрических) используют интервальную шкалу и обычные вещественные переменные.

Таким образом, цифровая форма представления информации в геоинформатике выполняет интегрирующую функцию и создает возможность совместной обработки геоданных полученных их разных источников.

Общие характеристики цифровых моделей. Развитие методов обработки информации привело к появлению нового направления в моделировании - цифрового моделирования и специальных моделей, называемых цифровыми моделями $[1,9]$. Важным 
в содержании «цифровой модели» является то, что она сформирована в цифровом коде, который воспринимает компьютер и может проводить обработку на этой основе.

Наибольшее распространение цифровые модели нашли в геоинформатике, проектировании, строительстве, архитектуре, экологии. В средине 80-х годов на страницах журнала геодезия и картография развернулась дискуссия о направлении развития цифровых моделей [10]. Сторонники первой концепции предлагали изменить существующее картографическое обеспечение под компьютерные технологии. Сторонники второй концепции предлагали адаптировать цифровые методы под картографические требования. Победила, и время это показало, вторая концепция. Она является основной в настоящее время.

В широком смысле цифровая модель $[1,8]$ это дискретная модель пространственных объектов, в которой одними из обязательных параметров являются: координаты, размеры, габариты, точность координат, масштаб и т.д. Естественно, что эта модель предназначена для обработки в информационных или геоинформационных технологиях.

В науках о Земле цифровая модель (ЦМ) это дискретная модель, сформированная для обработки пространственной информации с помощью компьютерных (геоинформационных) технологий. Среди пространственных цифровых моделей выделяют несколько видов: цифровая модель местности [11], цифровая модель объекта, цифровая модель явления (процесса). Наибольшее применение находит цифровая модель местности.

Цифровая модель объекта (ЦМО) - информационная дискретная модель объекта, предназначенная для хранения и обработки в компьютерных технологиях. Включает совокупность координат точек, характеризующих объект, связи между точками, информацию о свойствах объекта

Цифровая модель явления (ЦМЯ) или процесса - дискретная модель пространственного явления (процесса), предназначенная для хранения и обработки в компьютерных технологиях Ее особенностью является то, что она формируется в виде наборов данных, образующих временной ряд. Это дает возможность осуществлять мониторинг и осуществлять получение прогнозных оценок.

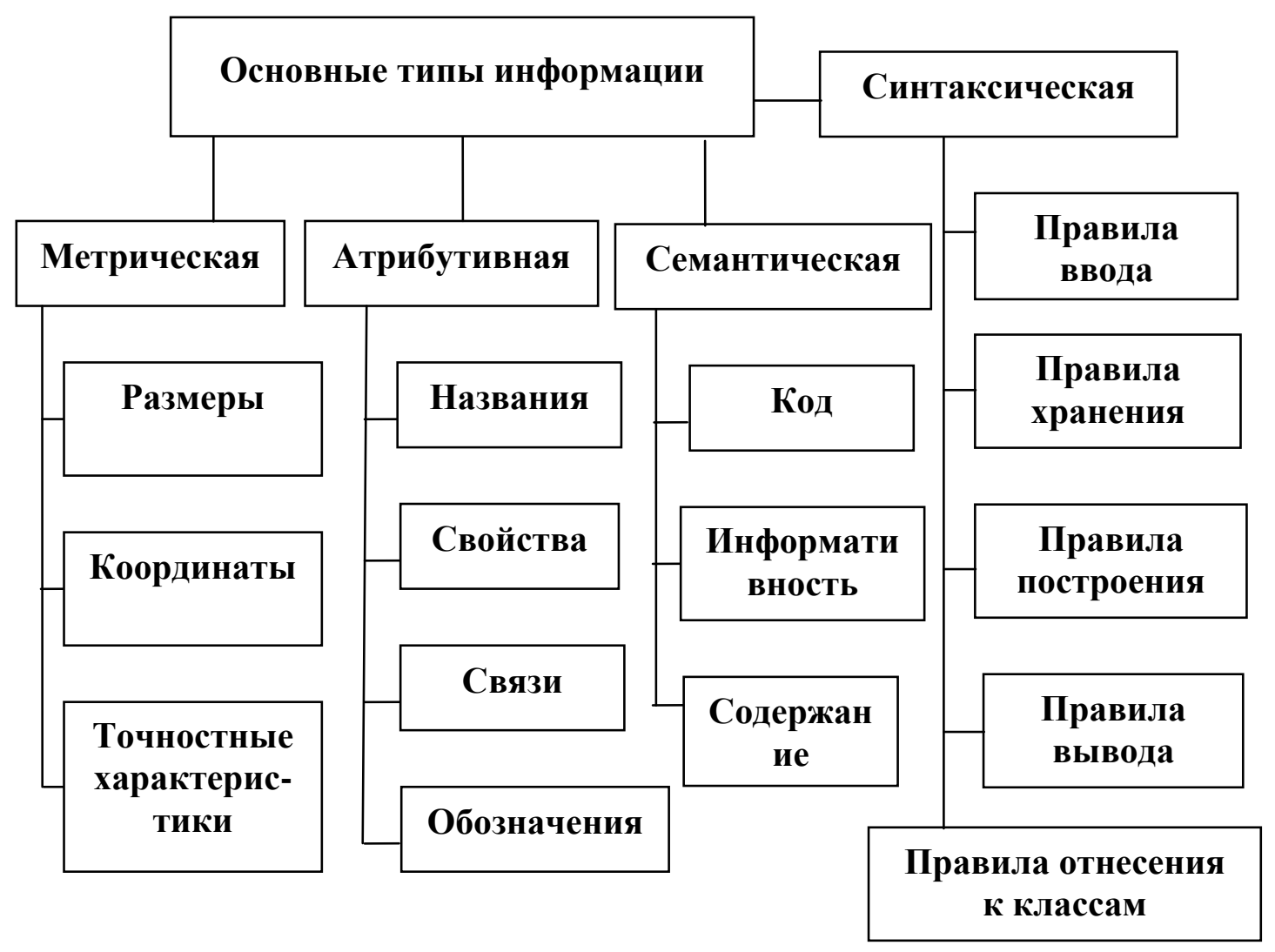

Puc. 1. Основные типы информации в цифровой модели 
Цифровые модели содержат различные типы информации [12]. Эти типы показаны на рис. 1. По аспекту пространственных отношений выделяют метрическую и атрибутивную типы информации.

По аспекту семиотического [13] анализа выделяют семантическую и синтаксическую составляющие.

Метрическая информация определяет положение путем задания абсолютных координат точек ЦММ и размеры объекта путем относительных координат точек в условных или местных системах. Качественным отличием метрической информации цифровых моделей, полученных по реальным измерениям, является точностная характеристика. Она обусловлена ошибками измерений и последующими ошибками вычислений. Этот параметр определяет применимость цифровой модели при решении практических задач в разных масштабах.

Атрибутивная информация определяет принадлежность точек или объектов к определенному классу или объекту [14]. Она описывает свойства объектов и их частей, задает взаимосвязи и условия обработки, условия воспроизведения и т.п. также как и во всей геоинформатике она решает главную задачу нахождения пространственных отношений

Семиотический аспект позволяет [13], рассматривая ЦМ как информационную модель, вводит известные в информатике оценки коэффициент информативности и коэффициент содержательности цифровой модели.

Семантическая часть информации [15] определяет ее содержательную сторону, она связана с кодированием данных. Синтаксическая информация определяет набор правил и отношений работы с цифровой моделью как с обычной информационной моделью. Она связана с классификацией и правилами построения моделей.

Аспект рассмотрения структуры цифровой модели позволяет выделить в ней логическую и физическую структуры [12] (рис.2).

Логическая структура модели [16] определяется как совокупность схем и логических записей, описывающих данную ЦМ. Она может включать схемы взаимосвязи частей ЦММ в натуре, в базе данных, схемы взаимосвязи свойств ЦММ и схемы построения ЦММ.

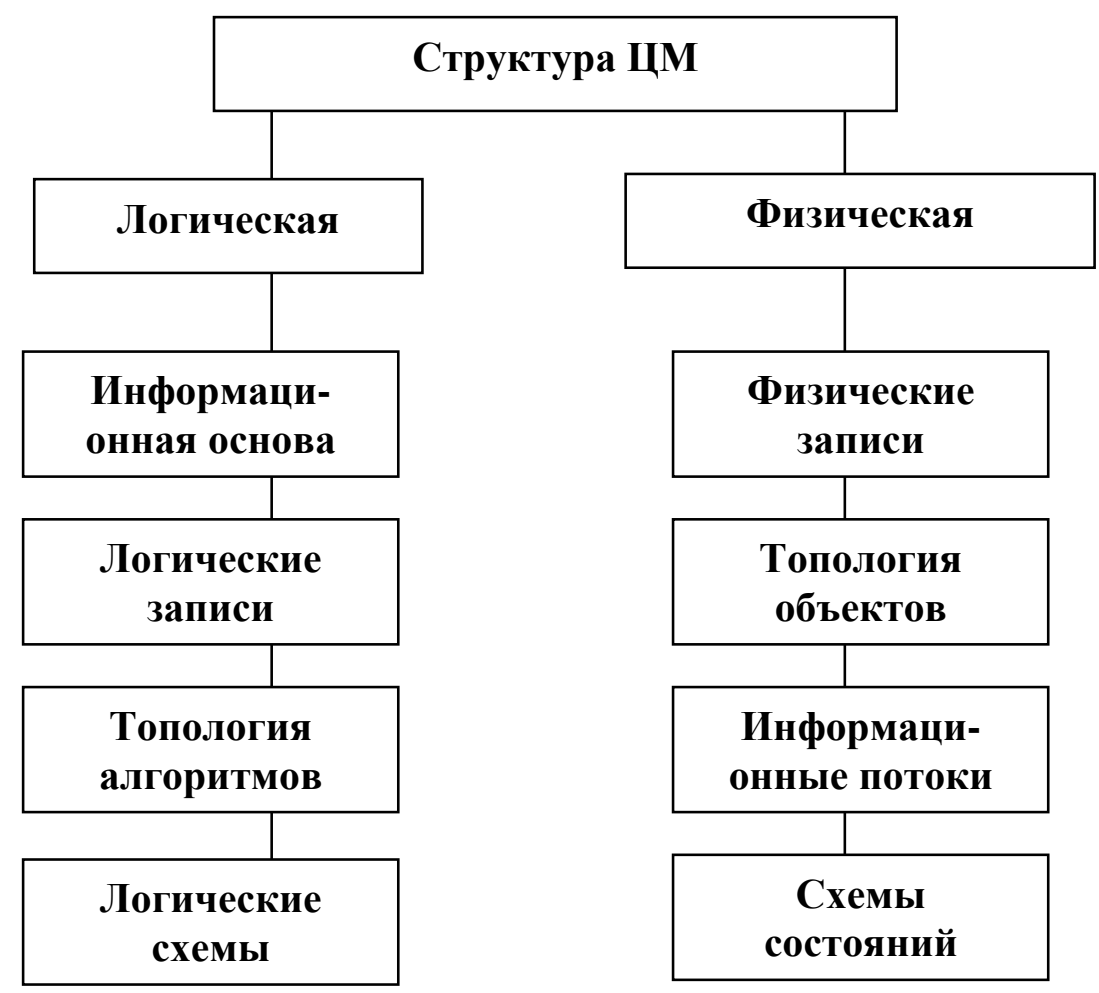

Puc. 2. Логическая и физическая структура ЦМ 
Она содержит логические записи, составляющие информационную основу. Элементом логической структуры ЦММ является логическая запись. Физическая структура ЦММ определяется способом реализации логической ЦММ на конкретной технической основе. Она задает формат записи данных, размеры кластеров, слов и т.д. Элементом физической структуры ЦММ является физическая запись.

Эти две части являются соответствием даталогической и физической моделей [16]. Таким образом, цифровая модель это компьютерно - ориентированная модель и модель, объединяющая даталогическую и физическую модели. Кроме того, цифровая модель должна быть стандартизована и отвечать требованиям стандартизации программных средств и продуктов [17].

Цифровая модель местности. Наибольшее применение в науках о Земле находит цифровая модель местности. Цифровая модель местности (ЦММ) (digital terrain model, $D T M$ ) отображает поверхность (а иногда и объекты местности) и содержит информацию о них, в виде в дискретных наборов данных.

Цифровая модель местности (ЦММ) $[11,12]$ - информационная дискретная модель местности, предназначенная для хранения и обработки в компьютерных технологиях. Она должна содержать следующую совокупность свойств: свойства модели местности, свойства информационной модели, семиотические свойства, свойства компьютерной модели, свойства моделей данных, свойства модели базы данных, свойства координатной модели, измеримость, потребительские свойства информационной продукции, свойства геоданных. Важным свойством современных цифровых моделей является учет пространственных отношений и геореференций [18]. ЦММ обладает следующими свойствами

1. Как модель местности ЦММ является описанием местности и объектов на ней. Она должна содержать специальную тематическую информацию о моделируемых объектах. Это означает, что ЦММ должна содержать описания и характеристики моделируемых объектов. Она должна выполнять описательную функцию объекта моделирования.

2. Как информационная модель ЦММ должна представлять объект моделирования в информационном поле, виде совокупности информационно определяемых параметров. Причем эти параметры должны описывать не только основные свойства объекта моделирования, но и основные отношения внутри объекта, а также между объектом и внешней средой.

3. Семиотические свойства ЦММ [13] состоят в том, что она должна содержать в своем описании три качественные семиотические характеристики:

- синтаксис - правила построения и использования;

- семантику - содержательную часть об объекте моделирования;

- прагматику - полезность и допускать меру оценки полезности

(ценности) от самой модели или от ее применения.

4. Как компьютерная модель ЦММ должна быть организована в виде структуры, позволяющей ее хранить и обрабатывать на компьютере. Это означает, что для полной ее реализации должна быть определена ее даталогическая и физическая составляющая. Она должна быть представлена в цифровом коде на машиночитаемом носителе информации.

5. Как модель данных вообще ЦММ должна быть определена на известном классе моделей. Это означает, что она должна в качестве логической основы содержать одну из базовых моделей данных, а также удовлетворять требованиям и обладать общими свойствами моделей соответствующего класса безотносительно к предметной области ее применения. Например, ее структура может быть сетевой, иерархической, линейной и т.п.

6. Как содержимое базы данных ЦММ должна быть организована не виде файловой системы, а структурирована в модели базы данных. Это накладывает на нее дополнительные 
условия типизации данных и сруктурирования ее частей. Это определяет аналогичные требования к семействам ЦММ для возможности их совместного хранения в БД.

7. Как координатная модель ЦММ должна обладать функциями метричности и координации. Это означает, что точки ЦММ определены в известной системе координат и выражены в определенных единицах измерений.

8. Как информационная продукция [19] ЦММ должна обладать потребительскими свойствами, делающими ее привлекательным товаром и обуславливающим спрос не нее как на коммерческую продукцию. В частности, должна быть обеспечена возможность ее применения для получения материальных благ. С целью повышения потребительской полезности ЦММ в базе данных должна быть информативно переопределена, чтобы ее можно было использовать для решения не одной, а различных задач.

9. Как форма представления геоданных ЦММ должна отражать и пространственно временные отношения, пространственные отношений и геореференции [18]. Она должна быть пригодна для решения задач картографирования, построения карт, включая цифровые карты.

В геоинформатике [12] процесс цифрования является процессом стратификации, то есть разбиением на уровни в соответствии с выбранными критериями. В этом случае величина уровней для разных объектов различна. На рис. 3 показан процесс получения цифровой модели в геоинформатике.

Исходный объект (рис. 3 а) представляет из себя непрерывное распределение некой величины «Н» от величины «L». Это может быть реальная высота (тогда речь пойдет о модели рельефа), это может быть уровень концентрации (или ПДК) химического вещества распределенного по территории, это может быть концентрация (например, населения) и т.д.

Процесс построения сводится к стратификации исходного объекта, то есть к разбиению непрерывной зависимости на дискретную. Уровни образуют «ступенчатую» модель рис. 3 б, которая по возможности должна соответствовать непрерывной.

Эта «ступенчатая» модель и называется цифровой. В дальнейшем именно она хранится в базе данных и подвергается обработке.

По данной цифровой модели местности, которая представляет набор дискретных точек $Z_{i}=f\left(x_{i}, y_{i}\right)$ можно строить непрерывные горизонтали $Y=\varphi(x)$ при $Z=$ const $_{\mathrm{i}}$ рис. 3 в

Цифровая модель может являться динамической характеристикой. Например, на ее основе осуществляется имитационное моделирование, позволяющее «проигрывать» упреждающие варианты развития оползневой ситуации при изменении внешних условий (сценарии развития) [20]. 


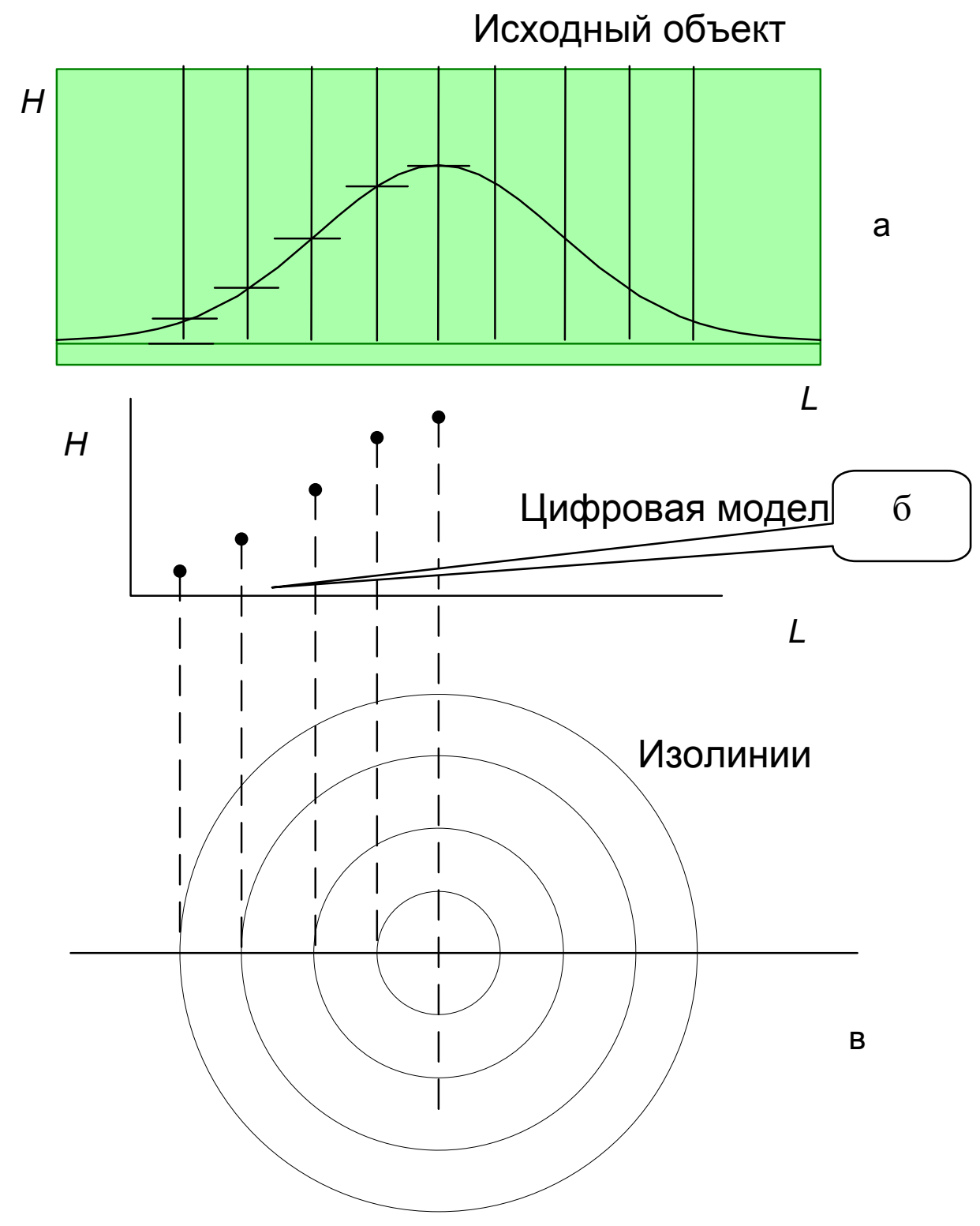

Puc. 3. Процесс построения и представления цифровой модели

\section{Заключение}

Цифровое моделирование решает важную когнитивную задачу преодоления семантического разрыва между потребностями и возможностями человека [21]. Цифровое моделирование в области наук о Земле имеет свою специфику. В радиотехнике цифровое моделирование связано с передачей сигналов. В науках о Земле цифровое моделирование связано с моделированием поверхности и пространственных объектов, для последующих расчетов и решения прикладных задач. Цифровое моделирование включает трехмерное моделирование объектов на поверхности земли и проведения разнообразных расчетов и выполнения проектов. Цифровые модели являются информационным ресурсом, имеющим государственное значение.

\section{Примечания:}

1. Heanue J. F., Bashaw M. C., Hesselink L. Volume holographic storage and retrieval of digital data // Science. 1994. T. 265. №. 5173. C. 749-752

2. Schroeder M. Number theory in science and communication: with applications in cryptography, physics, digital information, computing, and self-similarity. Springer, 2008. 
3. Toumazou C., Hughes J. B., Battersby N. C. (ed.). Switched-currents: an analogue technique for digital technology. - Iet, 1993. №. 5

4. Lee W. C. Y. Mobile cellular telecommunications: analog and digital systems. - McGrawHill Professional, 1995

5. Цветков В.Я. Глобализация и информатизация // Информационные технологии, 2005, №2. C. 2-4.

6. Майоров А.А., Цветков В.Я. Геоинформатика как важнейшее направление развития информатики // Информационные технологии. 2013. № 11. С. 2-7

7. Simavoryan S.Zh., Simonyan A.R., Ulitina E.I., Simonyan R.A. About one Approach to a Question of Classification of Intellectual Systems of Information Security // Modeling of Artificial Intelligence, 2014, Vol.(1), No 1, P. 29-44. DOI: 10.13187/mai.2014.1.29

8. Цветков В.Я. Клод Элвуд Шеннон, как основоположник цифрового моделирования // Перспективы науки и образования. 2014. №1. С. 44-50.

9. Майоров А.А., Цветков В.Я. Хранение и защита информационных ресурсов кадастра. М.: Московский государственный университет геодезии и картографии, 2009. 126 с.

10. Цветков В.Я. О двух концепциях автоматизации // Геодезия и картография. 1986. №5. C. 48-51.

11. Dietrich W. E. et al. Analysis of erosion thresholds, channel networks, and landscape morphology using a digital terrain model //The Journal of Geology. 1993. C. 259-278.

12. Иванников А.Д., Кулагин В.П., Тихонов А.Н. Цветков В.Я. Прикладная геоинформатика. М.: МаксПресс, 2005. 360 с.

13. Цветков В.Я. Семиотический подход к построению моделей данных в автоматизированных информационных системах // Геодезия и аэрофотосъемка, 2000, №5, c. $142-145$.

14. Heywood I., Cornelius S., Carver S. An Introduction to Geographical Information Systems, Pearson Education Limited, 2006. 426 p.

15. Tsvetkov V. Ya. Spatial Information Models // European Researcher, 2013, Vol.(60), № 10-1, p. 2386-2392.

16. Поляков А.А., Цветков В.Я. Прикладная информатика М.: Янус-К, 2002. 392 с.

17. Цветков В.Я. Стандартизация информационных программных средств и программных продуктов. М.: МГУГиК, 2000. 116 с.

18. Майоров А.А., Цветков В.Я. Геореференция как применение пространственных отношений в геоинформатике // Известия высших учебных заведений. Геодезия и аэрофотосъемка. 2012. №3. С. 87-89.

19. Цветков В.Я. Задачи геомаркетинга // Известия высших учебных заведений. Геодезия и аэрофотосъемка. 2000. №5. С. 146-154.

20. Скнарина Н. А. Роль и место физического и имитационного моделирования для оценки условий образования и динамики оползневых процессов // Вестник Ростовского государственного университета путей сообщения. 2011. №. 1. С. 23-27.

21. Tsvetkov, V.Ya. Information Interaction as a Mechanism of Semantic Gap Elimination // European Researcher, 2013, Vol.(45), № 4-1, p. 782-786

\section{References:}

1. Heanue J. F., Bashaw M. C., Hesselink L. Volume holographic storage and retrieval of digital data //Science. 1994. T. 265. №. 5173. P. 749-752

2. Schroeder M. Number theory in science and communication: with applications in cryptography, physics, digital information, computing, and self-similarity. - Springer, 2008.

3. Toumazou C., Hughes J. B., Battersby N. C. (ed.). Switched-currents: an analogue technique for digital technology. Iet, 1993. №. 5

4. Lee W. C. Y. Mobile cellular telecommunications: analog and digital systems. McGraw-Hill Professional, 1995

5. Tsvetkov V.Ya. Globalization and information // Information Technology. 2005. №2. P. 2-4.

6. Maiorov A.A Tsvetkov V.Ya. Geoinformatics as the most important area of computer science // Information Technology. 2013. № 11. P. 2-7.

7. Simavoryan S.Zh., Simonyan A.R., Ulitina E.I., Simonyan R.A. About one Approach to a Question of Classification of Intellectual Systems of Information Security // Modeling of Artificial 
Intelligence, 2014, Vol.(1), No 1, P. 29-44. DOI: 10.13187/mai.2014.1.29

8. Tsvetkov V.Ya. Claude Elwood Shannon, as the founder of digital simulation // prospects of science and education. 2014. №1. P. 44-50

9. Maiorov A.A., Tsvetkov V.Y. Storage and protection of information resources inventory. M.: Moscow State University of Geodesy and Cartography, 2009. 126 p.

P. 48-51

10. Tsvetkov V.Ya. Two concepts of automation // Geodesy and Cartography. 1986. №5.

11. Dietrich W. E. et al. Analysis of erosion thresholds, channel networks, and landscape morphology using a digital terrain model // The Journal of Geology. 1993. 6. P. 259-278.

12. Ivannikov A.D., Kulagin V.P., Tikhonov A.N., Tsvetkov V.Ya. Applied Geoinformatics. M.: MaksPress, 2005. $360 \mathrm{p}$.

13. Tsvetkov V.Ya. Semiotic approach to building data models in automated information systems // Geodesy and aerial photography, 2000, №5, p. 142-145

14. Heywood I., Cornelius S., Carver S. An Introduction to Geographical Information Systems, Pearson Education Limited, 2006. 426 p.

15. Tsvetkov V. Ya. Spatial Information Models // European Researcher, 2013, Vol.(60), № 10-1, p. 2386-2392

16. Polyakov A.A., Tsvetkov V.Ya. Applied Informatics. M.: Janus K, 2002. 392 p.

17. Tsvetkov V.Ya. Standardization of information software and software products. M.: MGUGiK. 2000. 116 p.

18. Maiorov A.A., Tsvetkov V.Ya. Georeference of how the use of spatial relations in geoinformatics // News of higher educational institutions. Surveying and aerial photography. 2012. №3. P. $87-89$

19. Tsvetkov V.Ya. Tasks geomarketing // News of higher educational institutions. Surveying and aerial photography, 2000, №5, P. 146-154

20. Sknarina N.A. Role of physical modeling and simulation to evaluate the conditions of the formation and dynamics of landslide processes // Herald of Rostov State University of Railway Transport. 2011. №. 1. P. 23-27.

21. Tsvetkov V.Ya. Information Interaction as a Mechanism of Semantic Gap Elimination // European Researcher, 2013, Vol.(45), № 4-1, P. 782- 786.

УДК 528; 004.9

\section{Цифровое моделирование в науках о Земле}

Александр Анатольевич Лобанов

Московский государственный технический университет радиотехники, электроники и автоматики (МГТУ МИРЭА), Российская Федерация

Кандидат технических наук, доцент

E-mail: cvj2@mail.ru

Аннотация. Дается анализ методов цифрового моделирования в области наук о Земле. Показано различие цифрового моделирования в радиосвязи и в науках о Земле. Показан интеграционный аспект цифровых моделей. Показаны преимущества цифровых моделей перед аналоговыми моделями. Показано, что цифровые модели являются дискретными. Отмечены свойства цифрового моделирования. Показана логическая структура цифровых моделей.

ключевые слова: Информация; данные; геоинформатика; модели; моделирование; цифровое моделирование; цифровые модели. 